\title{
Article
}

\section{Sunburn Protection by Sunscreen Sprays at Beach}

\author{
Hao Ou-Yang ${ }^{1, *}$ and Richard Bradley Rzendzian ${ }^{1,2}$ \\ 1 Johnson \& Johnson Consumer Inc. Skillman, NJ 08558, USA; rrzendzi@ITS.JNJ.com \\ 2 Ernest Mario School of Pharmacy, Rutgers University, New Brunswick, NJ 08901, USA \\ * Correspondence: houyan4@its.jnj.com; Tel.: +1-908-874-2722 \\ Academic Editors: Marisanna Centini and Cecilia Anselmi \\ Received: 16 February 2017; Accepted: 14 March 2017; Published: 18 March 2017
}

\begin{abstract}
Background: The efficacy of sunscreen is evaluated by SPF values, which are quantitatively determined in laboratories on the backs of human subjects according to a standardized procedure. However, SPF cannot be directly translated to sunburn protection under real-life situations because actual efficacy depends on various factors related to human behaviors and environmental conditions. This study clinically evaluated the efficacy of two sunscreen sprays (SPF 30 and SPF 70) under natural sunlight exposure on healthy subjects at the beach. Methods: Twenty subjects were divided into two cells for the two sunscreen sprays (SPF 70 and SPF 30) in a single-center, actual usage test. The primary endpoint of the study was sunburn protection on the dorsal arms and the secondary endpoint was protection on the face and neck. Subjects stayed at the beach for $4 \mathrm{~h}$ after application of the sunscreens with normal beach activities. Subjects' behavior at the beach, the amounts of sunscreen applied and reapplied, and environmental conditions were all recorded. Results: There was no significant sunburn for a majority of the subjects in either cell. However, neither sunscreen completely blocked the sunburn, especially the face/neck area. We found that the SPF 70 sunscreen was more effective than the SPF 30 sunscreen. Conclusion: Modern sunscreen sprays, applied liberally, are effective in providing sunburn protection for the body in a beach setting.
\end{abstract}

Keywords: erythema; swimming; re-application; exposed skin surface; natural sunlight

\section{Introduction}

Solar ultraviolet radiation is divided into UVB (295-320 nm) and UVA (320-400 nm). Although UVB is primarily responsible for the sunburn reaction in skin, both rays contribute to the risk of skin cancer and the signs of pre-mature skin aging. Sunscreens were originally developed to help prevent sunburn. The efficacy of sunscreen is primarily indicated by its sun protection factor (SPF), which is a ratio of solar energies required to produce erythema (defined as minimal erythema dose or MED) on protected skin relative to unprotected skin [1]. SPF testing is conducted under conditions prescribed by the FDA monograph or other standards.

While a precise SPF value can be determined in a laboratory setting, it is only useful as a parameter for relative comparisons for consumers. It does not directly translate to a safe sun exposure duration because the MED of an individual at a certain point is often unknown and environmental factors-including geographic location and associated solar conditions (time of the year, time of the day, local clouds and ozone conditions, elevation etc.) - are situation-dependent and ever-changing. In addition, people wearing sunscreen may participate in different activities during outdoor exposure. They may also apply and reapply differently, making the translation of the SPF value even more challenging in real life.

Many people apply sunscreens for outdoor recreational activities, but there are not many clinical studies designed to evaluate the effectiveness of modern sunscreens under real environmental conditions and actual use. We believe it is important for patients and health care professionals 
to understand how modern sunscreens perform in real life. This information can complement the label SPF and assist consumers and health care professionals in making informed, evidence-based decisions when choosing an appropriate sunscreen product. It can also help manufacturers to improve sunscreen formulations so that the product is more relevant and easy to use for consumers.

Not all sunscreens are created equal. Modern broad-spectrum recreational sunscreens offer excellent and durable protection against both UVA and UVB while resisting wash-off from water and sweat. Aerosol sprays are very popular among consumers because they are convenient to use and relatively quick to apply. However, there are not many studies documenting the actual usage behavior of sprays and clinical testing of modern sunscreen sprays on target populations at the beach is scarce.

\section{Materials and Methods}

The study included 20 healthy, adult subjects with Fitzpatrick skin type II-IV (eleven for skin type II, seven for type III and two for type IV). Subjects who displayed erythema or excessive at baseline were not included in the study and subjects with known dermatological conditions or had been allergic to sunscreen ingredients were also excluded. The study was conducted at Ormond Beach, Florida during peak summer season (July 2012). The study protocol and informed consent were approved by the Internal Review Board. Subjects were randomly assigned to one of the two cells utilizing either SPF 30 or SPF 70 sunscreen spray (Neutrogena Beach Defense, Los Angeles, CA, USA) on exposed skin. The subjects were blinded as to which SPF they used in the study. Both sunscreens are 80 min water resistance, broad-spectrum, and photostable. On Day 1, subjects reported for baseline screening and study enrollment. Total body surface area (BSA) was assessed for each subject by Mosteller method based on individual's height and weight [2].

Subjects reported to the study location in appropriate attire on Day 2. The skin area covered by swimwear was calculated for each subject and subtracted from BSA to obtain individual exposed skin area. The areas that were covered by cloth were assessed as follows: The bikini top was approximated as two triangles for both breasts, the tank top as cylinder. The bikini bottom was approximated as two triangles (front and back) while the shorts were approximated as three cylinders for two legs and a belt line. Subjects were clearly informed of label warnings and usage directions outlined in the FDA monograph including instructions about re-applications: Reapply at least every $2 \mathrm{~h}$, immediately after towel drying and after $80 \mathrm{~min}$ of swimming/sweating. Reapply generously to all exposed skin. They underwent an initial indoor clinical examination to ensure there is no sunburn at baseline. Fifteen minutes before sun exposure, a trained staff member applied the sunscreen spray to each individual, at $\sim 2 \mathrm{mg} / \mathrm{cm}^{2}$ to all exposed skin (except for face) without rub-in. Subjects self-applied sunscreen onto face by first spraying the product into hands and then spreading it uniformly. Subsequent product applications during the study period were determined and self-applied by the subjects. The frequency of re-applications was recorded and test sample containers were weighed after each use and at the completion of the study.

Subjects conducted normal activities including walking, reading, and swimming over the course of $4 \mathrm{~h}$ at the beach. They were not allowed to engage in strenuous physical activities and were kept well-hydrated. Umbrellas and canopies were provided when needed. The use of other sun protection measures such as hats and sunglasses were also permitted. Subjects filled out activity logs to document their activities at the beach and reapplication behaviors. Staff recorded time spent outside under direct sun, as well as the frequency and duration of water activities for each subject based on the activity log at the end of the study. Outdoor conditions including the temperature, humidity, and accumulative UV dosage (measured by solar meter PMA2100, Solar Light Company Inc., Glenside, PA, USA) were recorded throughout the day.

On Day 3, subjects were evaluated for sunburn based on the following four-point grading scale (0-no sunburn; 1-minimal redness; 2-slight to moderate redness; 3-clear sunburn and/or edema). The primary location for clinical examination was subject's dorsal forearm and secondary location was neck and face. 


\section{Results}

We estimated that the total exposed skin surface area ranged from 0.91 to $1.89 \mathrm{~m}^{2}$ and the average was $1.32 \mathrm{~m}^{2}$ for the subjects in our study. The mean and standard deviation of exposed BSA was $1.26 \pm 0.27 \mathrm{~m}^{2}$ for the SPF 70 cell and $1.38 \pm 0.32 \mathrm{~m}^{2}$ for the SPF 30 cell. The weather was mostly clear for the beach day. The temperature and relative humidity charts in Figure 1a present typical beach conditions: high temperature at around $32{ }^{\circ} \mathrm{C}\left(90{ }^{\circ} \mathrm{F}\right)$ and high humidity at around $70 \%-80 \%$ throughout the study duration (approximately 11 a.m. to 3 p.m.). The biologically calibrated UV intensity measurement showed 3-4 MED/h during this period and the cumulative UV dose reached over 14 MED after $4 \mathrm{~h}$.

Table 1 shows the minutes each cell spent under direct sun (mean and range), as well as the time and frequency of water activities. Both cells on average spent more than 200 min under the sun (including time spent in the water). One subject did not have any water activity while another subject spent more than $3 \mathrm{~h}$ in the water during the study. A majority of the subjects went into the water two to four times, totaling 15 to 70 min of water activity.

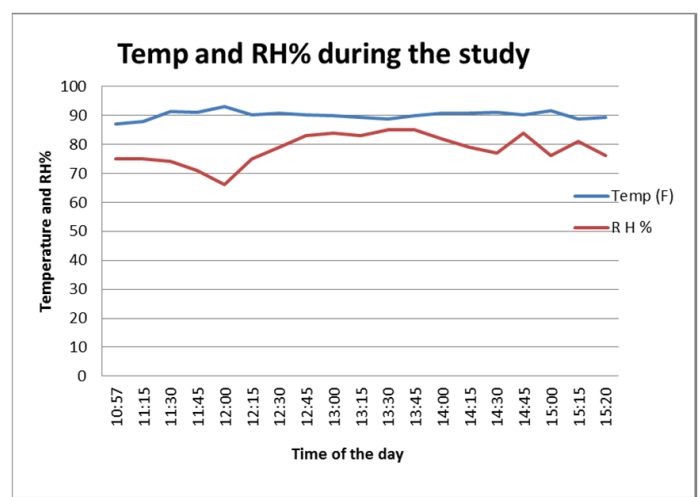

(a)

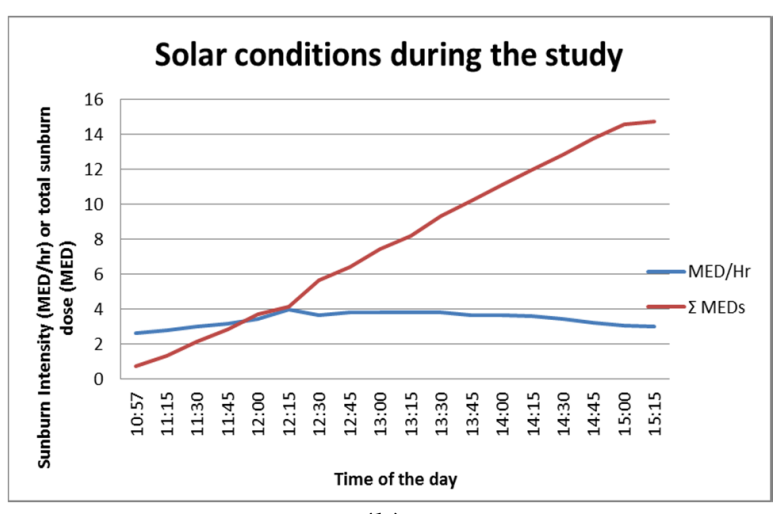

(b)

Figure 1. Environmental measures at beach. (a) Temperature and relative humidity during the study; (b) Solar conditions during the study. Blue line shows measured UV intensity (MED/h) and red line shows cumulative erythemal dose throughout the study.

Table 1. Subjects' sun exposure and water activity during the study. The brackets indicate measured ranges.

\begin{tabular}{cccc}
\hline Product Cell & Total Time Under Direct Sunlight (min) & Total Time in Water (min) & Number of Water Excursions \\
\hline SPF 70 & $221[189,240]$ & $37[13,123]$ & $3.1[1,5]$ \\
SPF 30 & $203[121,240]$ & $40[0,185]$ & $2.9[0,4]$ \\
\hline
\end{tabular}

Table 2 documents the amounts of sunscreen applied in this study. For both cells, more than $100 \mathrm{~g}$ of sunscreen was applied individually for the $4 \mathrm{~h}$ and subjects reapplied three to four times on average. The estimated initial surface density was $2.6 \pm 0.6 \mathrm{mg} / \mathrm{cm}^{2}$ for the SPF 70 cell and $2.3 \pm 0.5 \mathrm{mg} / \mathrm{cm}^{2}$ for the SPF 30 cell. The average overall surface density, defined as the total amount applied over the total numbers of application per area, was around $1.7 \mathrm{mg} / \mathrm{cm}^{2}$ for both cells. This clearly shows that subjects in this beach study applied sunscreen liberally and frequently. It also indicates that they may apply the sunscreen spray formulation more often than they do lotions/creams [3-5].

Table 2. Application amount and surface density (mean \pm standard deviation).

\begin{tabular}{|c|c|c|c|c|}
\hline Product Cell & $\begin{array}{c}\text { Number of } \\
\text { Applications }\end{array}$ & $\begin{array}{l}\text { Total Amount } \\
\text { Applied (g) }\end{array}$ & $\begin{array}{c}\text { Initial Surface } \\
\text { Density }\left(\mathrm{mg} / \mathrm{cm}^{2}\right)\end{array}$ & $\begin{array}{c}\text { Average Surface } \\
\text { Density }\left(\mathrm{mg} / \mathrm{cm}^{2}\right)\end{array}$ \\
\hline SPF 70 & $4.8 \pm 0.8$ & $108.2 \pm 79.7$ & $2.6 \pm 0.6$ & $1.7 \pm 0.7$ \\
\hline SPF30 & $4.3 \pm 1.3$ & $103.1 \pm 43.0$ & $2.3 \pm 0.5$ & $1.7 \pm 0.5$ \\
\hline
\end{tabular}


Clinical sunburn grading (Table 3) shows no clear erythema on the dorsal forearm on the following day except for one subject of skin type II in the SPF 30 cell. This was the subject who spent more than $3 \mathrm{~h}$ total in the water during exposure. This subject also had a clear sunburn on other body sites. The sunburn examination of the face/neck showed one case of minimal sunburn (score $=1$ ) in the SPF 70 cell and three cases (two cases of score $=1$ and one case of score $=2$ ) for the SPF 30 cell.

Table 3. Clinical sunburn grading based on a four-point scale.

\begin{tabular}{ccc}
\hline Product Cell and Site & $\begin{array}{c}\text { Observed Erythema Frequency } \\
\text { (\# of Subjects/Group) }\end{array}$ & Total Graded Score \\
\hline SPF 70-Dorsal Forearm & 0 of 10 & 0 \\
SPF 30-Dorsal Forearm & 1 of 10 & 2 \\
SPF 70-Face and Neck & 1 of 10 & 1 \\
SPF 30-Face and Neck & 3 of 10 & 4 \\
\hline
\end{tabular}

\section{Discussions}

It has been widely documented that consumers do not adequately apply and reapply cream and lotion sunscreen formulations. Insufficient application of sunscreen does not provide consumers with SPF protection as labeled on the product. Sunscreens with a higher SPF therefore may provide a margin of safety for consumers and patients [6]. With an easy-to-apply aerosol spray dosage form, consumers may be inclined to apply a higher dosage and reapply more frequently. This clinical study is among the first to confirm that modern, state-of-the-art sunscreen sprays provide excellent sunburn protection under actual-use conditions at beach.

Both cells (SPF 70 vs. SPF 30) were well balanced in terms of exposed skin areas, sun exposure duration, application amounts and beach activities. Both subjects and graders were blinded as to the SPF level of the products tested. Subjects were aware that they participated in a clinical study but their beach activities and reapplication behavior were autonomous. The reapplication data showed that people may use higher amounts of sunscreen spray when compared to a lotion. This may not come as a surprise since the sprays were designed to deliver a large amount of product to skin sites in a few seconds. This finding will need to be confirmed with additional studies including ones with a larger population. The study found that SPF 70 protected better than SPF 30 based on the total sunburn score of the forearm and face/neck (one vs. six). Currently, SPF 70 can only be labeled as SPF 50+ in the EU but can be labeled as SPF 70 in US as per the FDA. More studies should be conducted to confirm whether higher SPF products can benefit consumers in actual use settings.

The study results also indicate that neither sunscreen formulation completely prevents sunburn, particularly for the face/neck. It is known that people tend to apply less sunscreen to the face and sunscreen sprays need to be sprayed on the hand first and then rubbed into face/neck area. In any case, consumers should not solely rely on sunscreen. Instead, sun protection behaviors including wearing protective clothing, seeking shade and avoiding mid-day sun should be applied in conjunction with sunscreen.

An actual-use clinical study under real sunlight demonstrated that modern sunscreen sprays can effectively protect the body skin when applied liberally and reapplied frequently. Consumers and health care professionals can continue to use and recommend sunscreen sprays as part of an overall sun protection regimen.

Author Contributions: Hao Ou-Yang conceived and designed the experiments; Hao Ou-Yang helped perform the experiments; Hao Ou-Yang and Richard Bradley Rzendzian analyzed the data; Hao Ou-Yang and Richard Bradley Rzendzian wrote the paper.

Conflicts of Interest: The authors are employees of Johnson and Johnson who manufactures and commercializes the sunscreen tested in the study. 


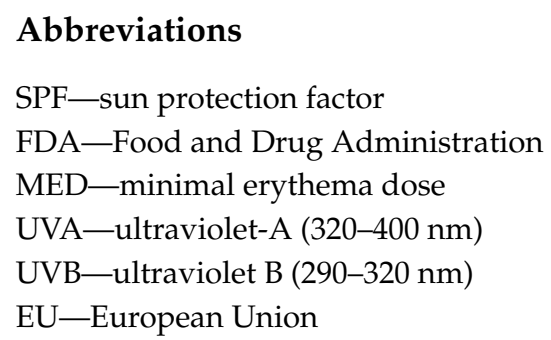

\section{References}

1. Food and Drug Administration. Labeling and Effectiveness Testing; Sunscreen Drug Products for Over-the-Counter Human Use; Final Rule. Fed. Regist. 2011, 76, 35620-35665.

2. Mosteller, R.D. Simplified calculation of body-surface area. N. Engl. J. Med. 1987, 317, 1098. [PubMed]

3. Bech-Thomsen, N.; Wulf, H.C. Sunbathers' application of sunscreen is probably inadequate to obtain the sun protection factor assigned to the preparation. Photodermatol. Photoimmunol. Photomed. 1992, 9, 242-244. [PubMed]

4. Robinson, J.K.; Rigel, D.S.; Amonette, R.A. Summertime sun protection used by adults and their children. J. Am. Acad. Dermatol. 2000, 42, 746-753. [CrossRef] [PubMed]

5. Lademann, J.; Schanzer, S.; Richter, H.; Pelchrzim, R.V.; Zastrow, L.; Golz, K.; Sterry, W. Sunscreen application at the beach. J. Cosmet. Dermatol. 2004, 3, 62-68. [CrossRef] [PubMed]

6. Ou-Yang, H.; Stanfield, J.; Cole, C.; Appa, Y.; Rigel, D. High-SPF sunscreens (SPF $\geq 70$ ) may provide ultraviolet protection above minimal recommended levels by adequately compensating for lower sunscreen user application amounts. J. Am. Acad. Dermatol. 2012, 67, 1220-1227. [CrossRef] [PubMed]

(C) 2017 by the authors. Licensee MDPI, Basel, Switzerland. This article is an open access article distributed under the terms and conditions of the Creative Commons Attribution (CC BY) license (http:/ / creativecommons.org/licenses/by/4.0/). 